Mots. Les langages du politique

70 | 2002

La politique en chansons

\title{
Jean Quéniart (dir.), Le chant acteur de l'histoire
}

\section{Marie-Anne Paveau}

\section{(2) OpenEdition}

\section{Journals}

\section{Édition électronique}

URL : https://journals.openedition.org/mots/9793

DOI : $10.4000 /$ mots.9793

ISSN : 1960-6001

\section{Éditeur}

ENS Éditions

\section{Édition imprimée}

Date de publication : 1 novembre 2002

Pagination : 141-143

ISBN : 2-84788-016-X

ISSN : 0243-6450

Référence électronique

Marie-Anne Paveau, " Jean Quéniart (dir.), Le chant acteur de l'histoire », Mots. Les langages du politique [En ligne], 70 | 2002, mis en ligne le 07 mai 2008, consulté le 22 avril 2022. URL : http:// journals.openedition.org/mots/9793; DOI : https://doi.org/10.4000/mots.9793

(c) ENS Éditions 
Jean QUÉNIART (dir.), Le chant acteur de l'histoire, Actes du colloque tenu à Rennes du 9 au 11 septembre 1998, Rennes, 1999, Presses universitaires de Rennes, 363 p.

Cet ouvrage réunit les communications présentées lors d'un colloque consacré à l'étude, dans une perspective historique, des « textes appuyés sur un support musical » (p. 9). Quatre axes de recherche ont guidé les travaux : l'organisation formelle des textes, les structures musicales, les phénomènes de transfert (par exemple du sacré au profane ou au politique) et les modes de diffusion et d'évolution des genres. Nous ne rendrons pas compte ici de l'ensemble des 23 articles de l'ouvrage, mais nous choisirons d'insister sur les spécificités de certains genres de chant, sur la fonction politique directe de certains textes musicaux et enfin sur leurs enjeux linguistiques.

Trois communications mettent l'accent sur la fonction politico-culturelle assumée par certains genres spécifiques. G. Durosoir, dans «L'allégorie de la renommée royale dans les airs de ballet au temps de Louis XIII » (p. 77-90), montre comment le message politique s'inscrit dans le langage musical. Elle donne en particulier une typologie intéressante des allégories récurrentes dans les ballets royaux de Louis XIII et détaille la façon dont le soleil devient progressivement le symbole de la puissance royale : on apprend qu'il est d'abord une figure chorégraphique appelée "Aimé de tous» (les chevaliers groupés autour de la figure royale) avant de devenir allégorie directe de la royauté dans les ballets de Louis XIV. L'auteure étudie également le Ballet des quatre monarchies chrestiennes, contenant le message politique transparent de la soumission de l'Italie, l'Espagne et l'Allemagne à la couronne de France. Autre genre à fonction politique examiné dans cet ouvrage : le noël. A. Keilhauer («Un modèle détourné : noël et satire politique au $18^{\mathrm{e}}$ siècle », 
p. 153-166) montre comment le noël est utilisé sous l'Ancien Régime à des fins satiriques : par transposition du sacré au profane, par glissement d'une origine populaire à une pratique mondaine, les formes du noël deviennent les supports d'une critique sévère de la Cour. Valorisant le modèle simple de la Sainte Famille, le noël satirique finit par contribuer au renforcement des valeurs bourgeoises. Dans un tout autre registre et à une autre époque, mais toujours autour de la notion de genre, L. TournèsFortin est l'auteur de « L'introduction avortée de la musique noire américaine dans la liturgie catholique française (1960-1970)» (p. 265-278). Il examine en particulier la naissance en 1966 et le destin ultérieur de la " gospel night », définie ainsi par le curé de Saint-Germain-des-prés en 1969 :

Je me suis rendu compte qu'il ne fallait pas commencer par faire des messes en jazz, mais par créer de nouvelles célébrations beaucoup plus libres où des gens, étrangers ou indifférents à notre liturgie même rénovée, retrouveraient la voix de la prière avec leurs gouts, leurs manières de s'exprimer, y compris le jazz. Nous avons appelé cette formule gospel night (p. 269).

Cette innovation cristallise les oppositions conjointes de la hiérarchie catholique et d'une partie des fidèles, et active la défense du chant grégorien et du latin dans la liturgie. L'auteur conclut sur la dimension politique de l'échec de cette tentative : l'Église n'a vraisemblablement pas su intégrer une culture populaire internationale, ou plutôt non-nationale.

La fonction politique des chants est directement abordée par trois articles de l'ouvrage. Le travail conjoint de C. Grasland et A. Keilhauer, « Conditions, enjeux et significations de la formation des grands chansonniers satiriques et historiques à Paris au début du $18^{\mathrm{e}}$ siècle » (p. 165-181) isole clairement trois fonctions des chansonniers (au sens de recueils de chansons politiques), importantes pour la vie sociale, politique et culturelle : une fonction «d'archivage de connaissances sur le passé » (p. 178), car le chansonnier constitue une source documentaire pour les mémorialistes par exemple; une fonction éducative, car le chansonnier peut être outil pédagogique pour l'éducation du Prince, comme le rapporte dans ses Mémoires Saint-Simon chargé de l'éducation du jeune Louis XV; une fonction «de plaisir et de sociabilité » (p. 178), car le chansonnier est aussi instrument de cohésion sociale et de convivialité. L'étude de V. Adoumié sur La Muette de Portici, opéra d'Auber sur un livret de Scribe, porte sur l'influence directe du chant sur les évènements historiques :

Son duo du second acte "Amour sacré de la patrie» est sans conteste le 
déclencheur de l'émeute du 25 aout 1830 [en Belgique] conduisant, en quelques jours, à l'émancipation du joug hollandais. Jamais, peut-être, une œuvre n'a été à ce point le catalyseur d'une situation politique conflictuelle. Dans ce cas précis, le chant devient, au sens le plus physique du terme, acteur de l'histoire immédiate (p. 242).

On a donc là selon l'auteur un véritable «art politique et politisé » (p. 248), dans la mesure où il déclenche l'histoire mais donne également lieu à des lectures politiques différentes selon les situations. Enfin, l'article de N. Dompnier, "Le rôle de la jeunesse dans la diffusion des hymnes sous le régime de Vichy » (p. 279-290) examine la fonction de socialisation intense assumée par la pratique collective des chants dans cette période : elle est parée des vertus prêtées au régime (discipline, cohésion et harmonie du groupe, équilibre) et favorise surtout la réussite des manifestations publiques, elles-mêmes lieux de l'endoctrinement et de la propagande. Ce qui fait dire à l'auteure qu'ils constituent de véritables « relais propagandistes » (p. 287), dont la cible privilégiée est la jeunesse.

Terminons par un article qui pose un problème de politique linguistique. F. Dartois-Lapeyre, dans « Le chant en langue étrangère et régionale dans l'opéra aux $17^{\mathrm{e}}$ et $18^{\mathrm{e}}$ siècles $》$ (p. 101-123), examine, à propos de la naissance de l'opéra français, la dimension politique importante du choix de la langue :

Du point de vue linguistique, l'opéra apparait comme un rempart et une fortification contre l'étranger, auquel n'est laissée qu'une place limitée : s'il peut séduire par quelques traits exotiques stéréotypés, il ne doit jamais nuire au triomphe du français, afin de respecter l'ordonnancement fixé par un pouvoir, toujours présenté triomphant. La domination politique s'exprime par la maitrise du chant français, mode d'expression de l'élite de la civilisation française (p. 121).

L'ampleur du volume et l'empan des thèmes abordés ne nous ont pas permis de mentionner tous les travaux. Mais on a là un ouvrage de référence sur la place et la fonction du chant dans l'histoire française, qui présente des corpus originaux et nouveaux, offrant autant de discours à arpenter de façon pluridisciplinaire, en musique. 